\title{
CORRIGENDUM
}

\section{Interaction between DRD2 and AKT1 genetic variations on risk of psychosis in cannabis users: a case-control study}

Marco Colizzi, Conrad lyegbe, John Powell, Giuseppe Blasi, Alessandro Bertolino, Robin M Murray and Marta Di Forti

npj Schizophrenia (2015) 1, Article number: 15049; doi:10.1038/npjschz.2015.49; published online 18 November 2015

Correction to: npj Schizophrenia (2015) 1, Article number: 15025. doi:10.1038/npjschz.2015.25

In the published version of this article, in Table 1, under the entry 'Cannabis use', some $N$ (\%) data were incorrect due to a minor miscalculation. The corrected table appears below. The authors regret the error.

\begin{tabular}{|c|c|c|c|c|}
\hline & \multicolumn{2}{|c|}{$P Z$} & \multicolumn{2}{|c|}{ Controls } \\
\hline & $\mathrm{N}$ & $\%$ & $\mathrm{~N}$ & $\%$ \\
\hline \multicolumn{5}{|l|}{ Cannabis use } \\
\hline Yes & 158 & 71.2 & 142 & 62.3 \\
\hline No risk allele & 34 & 21.5 & 40 & 28.2 \\
\hline One risk allele & 89 & 56.3 & 82 & 57.7 \\
\hline Two risk alleles & 35 & 22.2 & 20 & 14.1 \\
\hline Never & 64 & 28.8 & 86 & 37.7 \\
\hline No risk allele & 20 & 31.2 & 17 & 19.8 \\
\hline One risk allele & 35 & 54.7 & 51 & 59.3 \\
\hline Two risk alleles & 9 & 14.1 & 18 & 20.9 \\
\hline \multicolumn{5}{|l|}{ Frequency of use } \\
\hline Daily & 92 & 63.9 & 43 & 39.8 \\
\hline No risk allele & 19 & 20.7 & 12 & 27.9 \\
\hline One risk allele & 51 & 55.4 & 22 & 51.2 \\
\hline Two risk alleles & 22 & 23.9 & 9 & 20.9 \\
\hline Weekly or less & 52 & 36.1 & 65 & 60.2 \\
\hline No risk allele & 12 & 23.1 & 20 & 30.8 \\
\hline One risk allele & 29 & 55.7 & 37 & 56.9 \\
\hline Two risk alleles & 11 & 21.2 & 8 & 12.3 \\
\hline No details & 14 & & 34 & \\
\hline
\end{tabular}

